Article

\title{
Risk of Antimicrobial Resistant Non-Typhoidal Salmonella during Asymptomatic Infection Passage between Pet Dogs and Their Human Caregivers in Khon Kaen, Thailand
}

\author{
Xin Wu ${ }^{1,2}{ }^{-}$, Sunpetch Angkititrakul ${ }^{1}\left(\mathbb{D}\right.$, Allen L. Richards ${ }^{3}\left(\mathbb{D}\right.$, Chaiwat Pulsrikarn ${ }^{4}$, \\ Seri Khaengair ${ }^{1}$, Amphone Keosengthong ${ }^{5}$, Supatcharee Siriwong ${ }^{6}$ and Fanan Suksawat ${ }^{1, * \text { DD }}$ \\ 1 Ecotoxicology, Natural Resources and Environment Project, Khon Kaen University, Khon Kaen 40002, \\ Thailand; wuxinddl@hotmail.com (X.W.); sunpetch@kku.ac.th (S.A.); serkan@kku.ac.th (S.K.) \\ 2 College of Veterinary Medicine, Yunnan Agricultural University, Kunming 650051, China \\ 3 Department of Preventive Medicine and Biostatistics, Uniformed Services University of the Health Sciences, \\ Bethesda, MD 20814, USA; Allen.Richards@comcast.net \\ 4 National Institute of Health, Department of Medical Sciences, Ministry of Public Health, Nonthaburi 11000, \\ Thailand; chaiwat.p@dmsc.mail.go.th \\ 5 Department of Veterinary Medicine, Faculty of Agriculture, National University of Laos, Vientiane 7322, Lao \\ People's Democratic Republic; Keosengthong1990@gmail.com \\ 6 Synchrotron Light Research Institute (Public Organization), Nakhon Ratchasima 30000, Thailand; \\ supatcharee@slri.or.th \\ * Correspondence: sjirap@kku.ac.th; Tel.: +66-62-915-9366
}

Received: 10 July 2020; Accepted: 31 July 2020; Published: 4 August 2020

Abstract: To explore the risk of antimicrobial resistant (AMR) non-typhoidal Salmonella during asymptomatic infection passage between pet dogs and human caregivers in Khon Kaen, Thailand, one hundred forty paired fecal samples $(n=280)$ were obtained from companion dogs and their human caregivers, interviewed from 140 households during 2019-2020. The purified Salmonella isolates were serotype-identified and tested for antimicrobial resistance against ampicillin, ciprofloxacin, chloramphenicol, nalidixic acid, streptomycin, sulfamethoxazole-trimethoprim, and tetracycline. The homologous Salmonella isolate pairs (suggesting Salmonella infections may have been due to passage between each one of the pair, or derived from the same source) were subsequently characterized by serotype screening, pulsed field gel electrophoresis (PFGE), and Synchrotron Fourier transform infrared spectroscopy (SR-FTIR). The Salmonella prevalence observed in dogs, $12.86 \%$ (18/140), was not significantly different from that observed in humans, $17.86 \%$ (25/140) using McNemar's test. The AMR patterns (the patterns among the isolates of pet dogs and caregivers) and the serotypes (thirteen serotypes with 18 isolates from pet dogs plus thirteen serotypes with 25 isolates from humans) between pet dogs and humans were not significantly different using Pearson's chi-squared test. The homologous Salmonella isolates from the Salmonella-present households was $5.13 \%(2 / 39)$. This study demonstrated that the hypothesis that there is a high risk of Salmonella infection passage between dogs and humans with close contact in Khon Kaen is doubtful. Only 5.13\% of homologous Salmonella isolates from Salmonella-present households were found in Khon Kaen, Thailand, although the prevalence of Salmonella-positive samples, serotypes, and antimicrobial resistance patterns were quite similar among the two populations.

Keywords: antimicrobial resistance; non-typhoidal Salmonella; asymptomatic infection passage; pet dogs; humans 


\section{Introduction}

Antimicrobial resistance (AMR) has been a global problem for managing the health care of people and animals. AMR pathogens make the problem worse, because these pathogens can transmit AMR genes and cause infections in humans and animals [1]. The measures of transmissibility of AMR pathogens between animals and humans could provide information to discern an important route of how AMR pathogens get to humans. However, it is hard to ascertain the transmission direction (whether it is transmitted from animals to humans or from humans to animals). As such, an association investigation would be a better choice.

Salmonella species (non-typhoidal) are a leading cause of 550 million diarrheal diseases worldwide each year [2]. Additionally, AMR Salmonella infections makes the problem worse, as they can lead to treatment ineffectiveness, increasing the risk of blood infections [3], and the subsequent spread of AMR [4]. A wide variety of Salmonella (non-typhoidal) agents were isolated and reported from the food chain [5]. Unfortunately, there is a lack of credible data for pets as a source of salmonellosis, despite salmonellosis from pets being suggested as an important AMR transmission route [6]. Pet dogs may be infected with Salmonella from eating contaminated food and contact with infected feces or other infected animals. Subsequently, humans may become infected after coming into contact with a sick dog. One survey reported that of the 128 Salmonella infected patients, 107 (84\%) ill people had contacted with dogs prior to getting sick [7]. Several cross-sectional studies also reported that the risk of salmonellosis in humans is increased if their pet dogs had salmonellosis without overt clinical signs due to their close relationship [8,9]. However, despite data suggesting a significant Salmonella transmission route between pet dogs and humans, reliable quantitative data on AMR Salmonella transmission via the pet dog pathway are still lacking [10].

According to the World Health Organization (WHO) database [11], the Southeast Asia region has a high burden of non-typhoidal salmonellosis. The report indicates that the Southeast Asia region contributes $20.69 \%$ cases $(16.28$ million/78.7 million) and $26.93 \%$ deaths $(15,929 / 59,153)$ of the global non-typhoidal salmonellosis. Similarly, another research report estimated worldwide cases and deaths caused by non-typhoidal Salmonella at 93.8 million cases, with 155,000 deaths occurring each year, and that in Southeast Asia, 22.8 million cases and 37,600 death occur [5]. On the other hand, it has been reported that $11.6 \%$ of diarrheal and $13.2 \%$ of non-diarrheal dogs were infected by Salmonella in Northeastern Thailand [9]. The estimated dog population in Thailand is about 8.5 million [12]. Thus, there appears to be a high risk of Salmonella infection among pet dog sources. However, there has been no research reported on the association of AMR Salmonella between pet dogs and human care givers in Khon Kaen, in Northeast Thailand.

In order to investigate the association between Salmonella infections (and more importantly AMR Salmonella infections) between pet dogs and humans in Khon Kaen, Thailand, matched pair fecal samples of pet dogs and their caregivers were assessed. The paired fecal samples from pet dogs and their caregivers were investigated in Khon Kaen for the presence, serotype identity, and antimicrobial susceptibility of Salmonella. In addition, the quantitative presence of homologous Salmonella isolates among paired individuals (pet dogs and their human caregivers) was ascertained.

\section{Material and Methods}

\subsection{Sample Size Calculation}

The formula: $\mathrm{n}=(z)^{2} p(1-p) / d^{2}$ was applied to calculate the required sample size. In the formula, ' $n$ ' represents the required sample size; ' $z$ ' represents the level of confidence according to a standard normal distribution (for a level of confidence of 95\%, $z=1.96$ ); ' $p$ ' represents the expected prevalence, and ' $d$ ' represents allowable error (here, it was set at $5 \%$ ).

Approximately 5\% of people in Thailand are infected with Salmonella, as reported by Sirinavin [13]. The prevalence of Salmonella infected pet dogs is approximately 10\%, as reported by Polpakdee [9]. The required sample sizes for humans and pet dogs were determined to be 73 and 138, respectively, 
according to the expected prevalence and allowable error. For paired dogs and humans, there was no preliminary study for Salmonella prevalence between the two populations. For this study, the required paired sample size was set according to the required pet dog sample size because it was larger than the human sample size required.

\subsection{Sample Collection}

One hundred and forty households were randomly interviewed from five villages (31 households from Bua Simma village, 24 from Mo Sum Soong village, 29 from Non Udom village, 44 from Noun Kung Soen village, and 12 from Nai Muang village) in Khon Kaen Province, Thailand, from 2019 to 2020 (random sampling). For the 140 household interviews, one pet dog and its closest caregiver were requested for sampling in pairs $(n=280)$. Contact means physical contact, and closest means the human caregiver who most often fed and cleaned the pet within the household. All subjects (both pet dogs and humans) were investigated for their history of antimicrobial usage for the past year. However, the history of antimicrobial usage was often incomplete, because many human volunteers could not provide information on the types of antimicrobials used. All samples were collected from asymptomatic individuals (both pet dogs and humans). The carriage of Salmonella among the individuals evaluated from the five villages were sporadic without apparent outbreak associations (i.e., no enteritis in groups occurred within the five villages, as indicated by the village health administrator). Fecal samples were collected using sterile rectal swabs for pet dogs and $10 \mathrm{~g}$ of feces using sterile plastic containers for human caregivers. All samples were matched and transported in an icebox. Salmonella isolation was subsequently performed at the laboratory. The study was guided and approved by the Institutional Animal Care and Use Committee of Khon Kaen University, No. IACUC-KKU-98/61, and Khon Kaen University Ethics Committee for Human Research, No. HE602286.

\subsection{Salmonella Isolation}

All fecal samples were immediately processed after transport for Salmonella isolation following the International Organization for Standardization (ISO) standard 6579-1:2002 at the Khon Kaen University Veterinary Teaching Hospital laboratory [14]. All swab samples were pre-enriched in $9 \mathrm{~mL}$ buffered peptone water (BPW) (HiMedia Laboratories, Mumbai, Maharashtra, India) at $37^{\circ} \mathrm{C}$ for $24 \mathrm{~h}$, and the fecal samples were transferred by swabs into $9 \mathrm{~mL}$ BPW. Then, each aliquot of $0.1 \mathrm{~mL}$ was selectively enriched on Modified Semisolid Rappaport Vassiliadis Medium plates (Becton Dickinson, MSRV, Franklin Lake, NJ, USA) at $42{ }^{\circ} \mathrm{C}$ for $24 \mathrm{~h}$. The turbid-grey material from MSRV was streaked onto xylose lysine deoxycholate agar (XLD) plates (Becton Dickinson) at $37^{\circ} \mathrm{C}$ for $24 \mathrm{~h}$, and secondly selected on Hektoen Enteric agar (HE) plates (Becton Dickinson) at $37^{\circ} \mathrm{C}$ for $24 \mathrm{~h}$. The presumptive colonies were identified as Salmonella by their black color.

\subsection{Salmonella Serotype Identification}

Three presumptive colonies from selective media with each sample were selected and assessed to determine their serotype(s). If the three colonies were of the same serotype, they would be regarded as one isolate. If different serotypes from the three presumptive colonies from an individual sample were identified, then all unique colonies were assessed and recorded. All presumptive colonies were biochemically identified by Triple Sugar Iron agar (TSI) (Becton Dickinson) and Motility Indole Lysine (MIL) medium (Becton Dickinson). The $\mathrm{O}$ (somatic) and $\mathrm{H}$ (flagellar) antigens of all isolates were characterized by agglutination with hyperimmune sera (S\&A Reagents Lab Ltd., Bangkok, Thailand), at the WHO National Salmonella and Shigella Center for serotype identification. The serotypes were assigned according to the Kauffmann-White scheme [15].

\subsection{Antimicrobial Susceptibility Testing}

The Salmonella isolates were analyzed for antimicrobial susceptibility to ampicillin (AMP) $10 \mu \mathrm{g}$, ciprofloxacin (CIP) $5 \mu \mathrm{g}$, chloramphenicol (CHL) $30 \mu \mathrm{g}$, nalidixic acid (NA) $30 \mu \mathrm{g}$, streptomycin (STR) 
$10 \mu \mathrm{g}$, sulfamethoxazole-trimethoprim (SXT) $25 \mu \mathrm{g}$ and tetracycline (TET) $30 \mu \mathrm{g}$ by the disk diffusion method (BD Diagnostics, Sparks, MD, USA), following the Clinical and Laboratory Standards Institute guidelines [16].

\subsection{The Statistical Analysis of Salmonella Asymptomatic Infection Passage between Paired Dogs and Their Caregivers}

To assay the epidemiological relatedness of Salmonella isolates from asymptomatic infection passage between the two populations, the presence of Salmonella-positive samples was statistically analyzed by McNemar's test. The serotypes and antimicrobial patterns of Salmonella isolates were statistically analyzed by Pearson's chi-squared test. The statistical analysis was performed using statistical software SPSS version 17.0 (SPSS Inc, Chicago, IL, USA), and $p<0.05$ was considered significant.

\subsection{The Determination of Homologous Salmonella Isolates}

To determine the homologous Salmonella isolate pairs, serotypes of all pet dog and human source isolates were screened for serotype-matched pairs of isolates (the isolates from a pet dog and its human caregiver belonging to the same serotype). Then, the serotype-matched pairs of isolates were tested by pulsed field gel electrophoresis (PFGE) and further confirmed by Synchrotron Fourier transform infrared spectroscopy (SR-FTIR) for homologous Salmonella isolate pairs. FTIR is a rapid and accurate detection technique, providing reliable data for bacterial strain level epidemiological research [17,18]. The detailed assessment of homologous Salmonella isolate pairs allowed for the demonstration of whether or not the AMR Salmonella spp. infected dogs and the infected humans of the same household were related (whether or not the infections arise from the passage between each other or from the same source).

The PFGE was performed following the Centers for Disease Control and Prevention (CDC) standard [19]. DNA fragments were digested by Xbal and separated by a CHEF-DRIII Pulsed-Field Electrophoresis System. The gel was stained with ethidium bromide and documented using a ChemiDoc $^{\mathrm{TM}}$ XRS+ (Bio-Rad, Hercules, California, USA). The dendrogram was produced using band clustering with a dice coefficient similarity index of $1 \%$ optimization and $1 \%$ tolerance by the unweighted pair group method with arithmetic means (UPGMA) by BioNumerics version 7.6 (Applied Maths, Keistraat, Sint-Martens-Latem, Belgium).

To prepare the tests for FTIR, the PFGE confirmed isolates were performed as follows: The Salmonella isolates were cultured in nutrient broth for $24 \mathrm{~h}$ at $28{ }^{\circ} \mathrm{C}$, and then the culture was centrifuged at $6000 \mathrm{rpm}$ for $5 \mathrm{~min}$. Cell pellets were washed with $0.85 \% \mathrm{NaCl}$, then re-centrifuged. The culture was dissolved in sterile distilled water and deposited into a $\mathrm{BaF}_{2}$ window. The prepared test windows were scanned by SR-FTIR microspectroscopy at the Synchrotron Light Research Institute (Public Organization), Thailand. The infrared spectra were collected using a Bruker IR spectrometer (VERTEX70) coupled to an IR microscope (Hyperion 2000 IR microscope coupled with VERTEX70 spectrometer, Bruker Optics, Ettlingen, Germany). Spectrum acquisition from 4000-600 $\mathrm{cm}^{-1}$ with transmission mode were collected by the OPUS 7.5 software (Bruker Optics Inc., Billerica, MA, USA) with a 36× objective lens, a background scan time of 64 scans and a resolution of $4 \mathrm{~cm}^{-1}$. Principal component analysis (PCA) and soft independent modelling of class analogy (SIMCA) were analyzed by the Unscrambler X 10.5 software (CAMO Software Inc., Newfoundland, Canada). All spectra of each test were transformed by second derivative and vector normalization in the range from $3000-2800 \mathrm{~cm}^{-1}$ and $1750-950 \mathrm{~cm}^{-1}$, according to principal component 1 (most principal component contributing for clustering, PC1). The PCA was used to classify based on the differentiation of the biochemical components in the whole cells from each strain. The SIMCA was used for the classification of spectrum data, which requires a training data set to identify the class membership. The training data set was from the spectra of the PFGE-matched isolates from dogs. The test data were the spectra of the PFGE-matched isolates from humans. The class memberships were shown by the percentage of correctly identified spectra data by SIMCA analysis. 


\section{Results}

\subsection{The Prevalence and AMR of Salmonella from Pet Dogs and Humans in Khon Kaen, Thailand}

The prevalence of Salmonella among dogs and humans was 12.86\% (18/140) and 17.86\% (25/140), respectively. Of all the positive samples, 13 Salmonella serotypes from 18 pet dog isolates were identified, and 13 serotypes from 25 human isolates were identified. The dominant serotypes (more than 10\%) detected from dogs were S. enterica serotype Stanley (16.67\%), S. enterica serotype Hvittingfoss (16.67\%), and S. enterica serotype I 1,4,[5],12:i:- (11.20\%). The dominant serotypes identified from humans were S. enterica serotype Stanley $(24.00 \%)$, S. enterica serotype Weltevreden $(16.00 \%)$, and S. enterica serotype I 1,4,[5],12:i:- (12.00\%) (Figure 1).

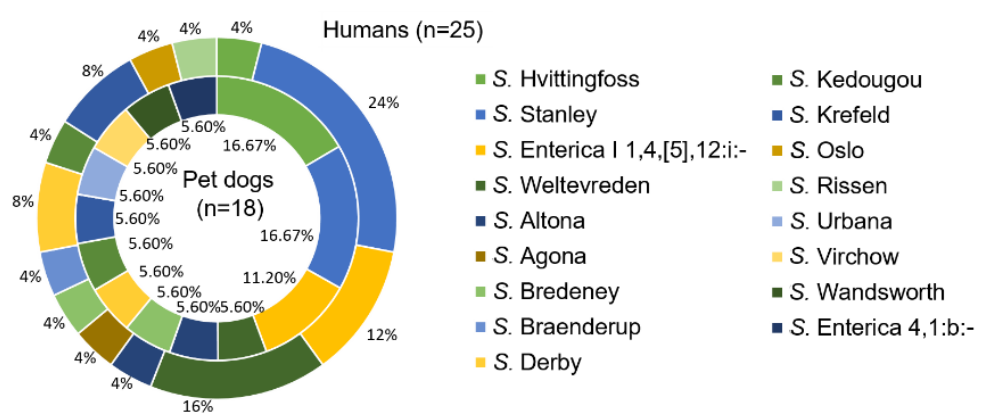

Figure 1. The serotypes of Salmonella from pet dogs and humans in Khon Kaen, Thailand. Note: thirteen serotypes from 18 Salmonella isolates were isolated from pet dogs, and 13 serotypes with 25 Salmonella isolates were from humans. The inner circle denotes the serotypes from pet dog source isolates. The outer circle denotes serotypes from human source isolates. The colors of the two circles denote different Salmonella serotypes.

Antimicrobial susceptibility testing of Salmonella among dogs and human caregivers determined antimicrobial resistance to: ampicillin (AMP) (44.44\% and 64.00\%), chloramphenicol (CHL) (16.67\% and $24.00 \%$ ), ciprofloxacin (CIP) (5.56\% and 12.00\%), nalidixic acid (NA) (5.56\% and 12.00\%), streptomycin (STR) $(22.22 \%$ and $32.00 \%)$, sulfamethoxazole-trimethoprim (SXT) (16.67\% and 24.00\%) and tetracycline (TET) $(38.89 \%$ and $64.00 \%)$, respectively. The total AMR containing Salmonella isolates from pet dogs was $44.44 \%(8 / 18)$, and $64.00 \%(16 / 25)$ in human caregivers.

\subsection{The Epidemiological Relatedness of Salmonella Infection among Paired Dogs and Humans}

The Salmonella species were identified from 43 individuals of 39 households (dogs only $(n=14)$, humans only $(n=21)$, both dogs $(n=4)$ and humans $(n=4))$, whereas 101 households were Salmonella negative. The tally of Salmonella infected dogs and humans, from paired data, is shown in Table 1. The relationship between pet dogs and their human caregivers for the occurrence of Salmonella infection was not statistically different $(p=0.310, n=140)$ when analyzed by McNemar's test. Thus, the Salmonella presence between pet dogs and humans was similar to each other.

Table 1. Tally of Salmonella-positive samples from pet dogs and their caregivers.

\begin{tabular}{cccc}
\hline \multirow{2}{*}{ Pet dogs } & \multicolumn{2}{c}{ Human Caregivers } \\
\cline { 3 - 4 } & & Positive & Negative \\
\hline \multirow{2}{*}{ Positive } & 4 & 14 \\
& Negative & 21 & 101 \\
\hline
\end{tabular}

Note: the tally of Salmonella-positive samples was sorted to assess the dependence of these paired samples using McNemar's test. The statistical difference of McNemar's test $(p<0.05)$ reveals that the presence of Salmonella among paired samples was independent of each other. 
Of the serotypes, nine serotypes (including S. enterica: Altona, Bredeney, Derby, Hvittingfoss, type I 1,4,[5],12:i:-, Kedougou, Krefeld, Stanley, and Weltevreden) were shared between the isolates of pet dogs and humans. The mutual serotype isolates in pet dogs were $77.78 \%(14 / 18)$, and 84.00\% (21/25) in humans (Figure 2). The serotypes of the Salmonella isolates from pet dogs and humans were not significantly different $(p>0.05)$, indicating the serotypes among pet dogs are quite similar to humans.

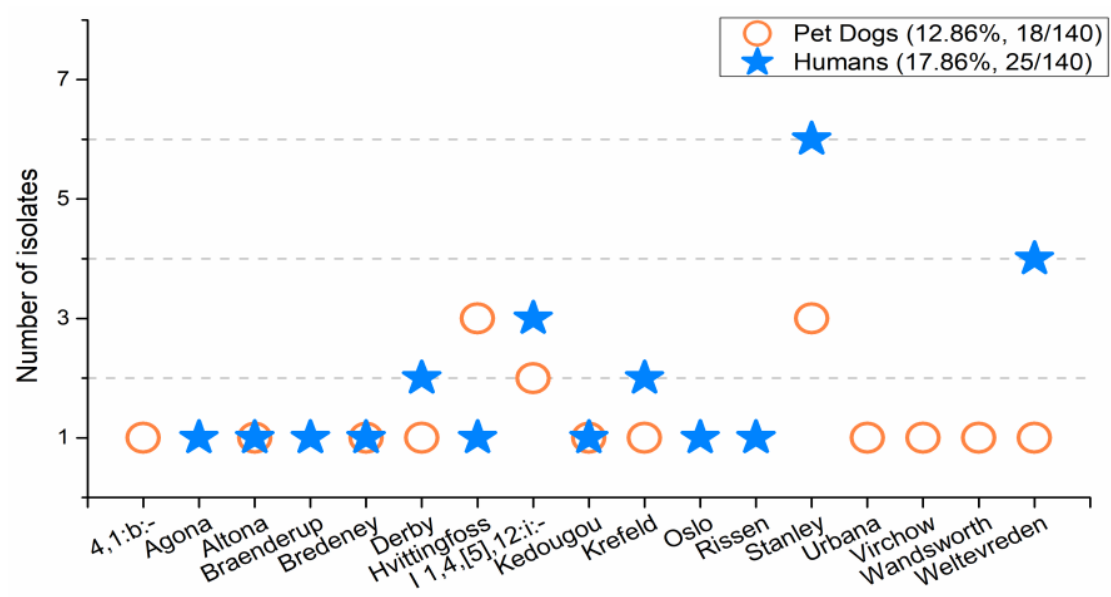

Figure 2. The serotypes of Salmonella from paired pet dogs and humans in Khon Kaen, Thailand. Note: the serotypes of pet dog fecal samples were quite similar to the serotypes from humans. Thirteen Salmonella serotypes were identified from pet dogs, and thirteen serotypes were from humans.

For the prevalence of AMR patterns, the AMR patterns of Salmonella isolates from the paired samples were shown in Table 2. The isolates of AMR patterns between the pet dog source and the human source were statistically analyzed using Pearson's chi-squared test. The resistance patterns of Salmonella isolates were not significantly different between human and pet dog isolates $(p>0.05)$. Thus, this demonstrates that the AMR patterns of the isolates from the two populations among pet dogs and human caregivers were similar.

Table 2. Antimicrobial resistance (AMR) patterns of Salmonella from pet dogs and their caregivers.

\begin{tabular}{lcc}
\hline \multicolumn{1}{c}{ Antimicrobial Resistance Patterns } & Isolate Numbers from Pet Dogs & Isolate Numbers from Humans \\
\hline AMP & 1 & 0 \\
AMP-TET & 2 & 4 \\
AMP-STR & 0 & 1 \\
CHL-TET & 0 & 1 \\
AMP-STR-TET & 2 & 6 \\
AMP-CHL-SXT-TET & 1 & 1 \\
AMP-CHL-STR-SXT-TET & 1 & 1 \\
AMP-CHL-CIP-NA-SXT-TET & 0 & 2 \\
AMP-CHL-CIP-NA-STR-SXT-TET & 1 & 1 \\
Sensitive to all antimicrobials & 10 & 8 \\
\hline
\end{tabular}

Note: AMP: ampicillin, CHL: chloramphenicol, CIP: ciprofloxacin, NA: nalidixic acid, STR: streptomycin, SXT: sulfamethoxazole-trimethoprim, TET: tetracycline. The AMR patterns of the isolates from the two populations were not significantly different $(p>0.05)$ using Pearson's chi-squared test. The antimicrobial-sensitive isolates were not considered in the statistical analysis.

After the determination of homologous Salmonella isolates among paired individuals (serotype screening, and confirmed by PFGE and SR-FTIR), two serotype-matched pairs, H121 and D121, and H86 and D86, were found to be homologous isolates (Figure 3). The 18 pet dog isolates and 25 human isolates were screened according to the serotype, shown by Venn diagram (Figure 3A). The two-paired serotype matched isolates (H121 and D121, and H86 and D86) were at the 100\% similarity level, which was confirmed by the PFGE method (Figure 3B). The PFGE-matched isolates were further confirmed by 
SR-FTIR and analyzed using PCA and SIMCA. All spectra of each test were transformed by second derivative and vector normalization in the range from $3000-2800 \mathrm{~cm}^{-1}$ and $1750-950 \mathrm{~cm}^{-1}$, according to principal component 1 (PC1). The loading PC1 (most component contributing for clustering) was introduced to adjust the best-distinguished range of spectra for further analysis (PCA and SIMCA), which explained the distinguished peak position. The positive loading PC1 showed that 1739, 1650, 1540 , and $1240 \mathrm{~cm}^{-1}$ were the distinguishing peaks from the negative side of the PCA score plot (H121 and D121 were grouped into the left side of PCA score plot). The negative side of the loading plot separated D86 and H86, and corresponded to distinguished peaks at 977, 1664, and $1641 \mathrm{~cm}^{-1}$. Therefore, the distinguished range of spectra was set at $3000-2800 \mathrm{~cm}^{-1}$ and $1750-950 \mathrm{~cm}^{-1}$ (Figure 3C). The PCA score plot separated the spectra of isolate pair H121 and D121 from H86 and D86 (Figure 3D). The class memberships analyzed by SIMCA were shown by the percentage of correctly identified spectra data. The percentage of correctly identified spectra of H121 by model-D121 was $96.72 \%$, whereas the percentage of model-D86 was $0.82 \%$. This indicated that the class membership of isolate H121 belongs to model-D121. The percentage of correctly identified spectra of H86 by model-D86 was $95.92 \%$, whereas the percentage of model-D121 was $30.61 \%$. This indicated the class membership of isolate H86 belongs to model-D86 (Figure 3E). The results of PCA and SIMCA revealed that the spectra of isolates H121 and D121 were the same, as for isolates H86 and D86 (Figure 3D,E). The homologous Salmonella isolate pairs indicate that the corresponding pet dog and human pairs were infected with the same Salmonella, either from passage between each other or the Salmonella was obtained individually from the same source (i.e., the same food-borne source or same infection source). The prevalence of homologous Salmonella pairs from the total Salmonella-present households was 5.13\% (2/39).

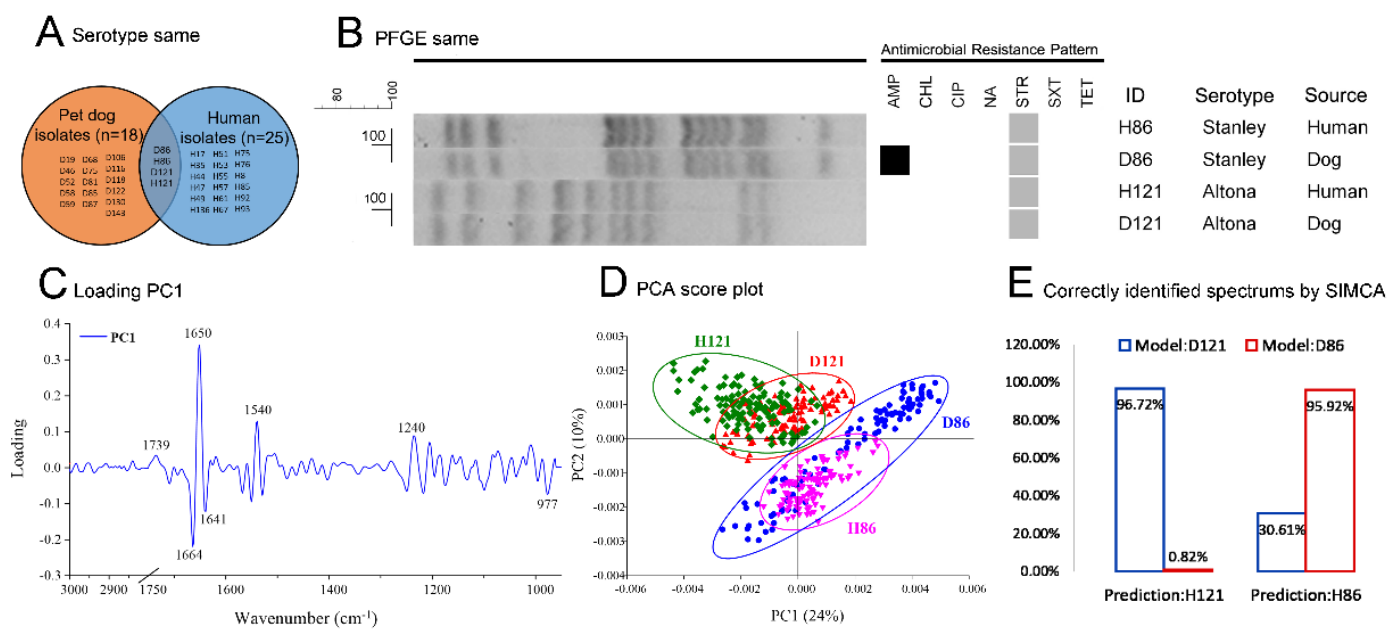

Figure 3. The determination of homologous Salmonella isolate pairs ( $\mathbf{\square}=$ resistance, $\square=$ intermediate). Note: (A) serotype screening result, shown by Venn diagram. Two pair isolates were serotype matched (H121 and D121, and H86 and D86). The code behind ' $\mathrm{H}$ ' or ' $\mathrm{D}$ ' denotes the household number (i.e., H86 and D86 are from the same household). (B) The isolates H121 and D121, and H86 and D86 exhibited a 100\% similarity level as confirmed by the pulsed field gel electrophoresis (PFGE) method. (C) Loading principal component 1 (PC1) result, the most principal component contributes for clustering. (D) Principal component analysis (PCA) result. (E) Soft independent modelling of class analogy (SIMCA) result. (C-E) The PFGE matched isolates were further confirmed by Synchrotron Fourier transform infrared spectroscopy (SR-FTIR) and analyzed using PCA and SIMCA. The results of the PCA and SIMCA revealed that the spectra of isolates H121 and D121 were the same, as for H86 and D86.

\section{Discussion}

The prevalence of Salmonella in pet dogs determined herein was $12.86 \%$, which was similar to that reported in a previous investigation in Thailand (13.2\%) [9], and in Ethiopia (11.7\%) [20], while it was 
much higher than that reported in the USA (2.5\%) [21]. The Salmonella prevalence of $17.86 \%$ in humans was higher than the $4.7 \%$ reported in Thailand [13]. As for the most predominant serotypes, S. enterica Stanley was one of the most common serotypes among dogs reported in Thailand [13]. Moreover, it was one of the ten most common S. enterica serotypes from Thai patients, along with Weltevreden and I 1,4,[5],12:i:- [22]. In Northeast Thailand, the S. enterica serotype Hvittingfoss was one of the top ten most common Salmonella serotypes detected [22]. Thus, the prevalence of Salmonella from pet dogs and humans in this study is consistent with other reports, and the serotypes detected herein are among the predominant serotypes reported in Thailand.

The prevalence of antimicrobial resistant isolates from pet dogs in this study (44.44\%) is lower than the reports from dogs in Addis Ababa, Ethiopia (90.5\%) [20], while it is similar to the report of dogs from Khon Kaen, Thailand (54.8\%) [9]. The prevalence of antimicrobial resistant Salmonella isolates from human caregivers in this study (64.00\%) was lower than human patients of $100 \%$ in Khon Kaen, Thailand [23]. The most prevalent antimicrobial resistant agents included ampicillin and tetracycline. The susceptibility patterns of this study correspond to other reports in Thailand for ampicillin, streptomycin, and tetracycline [24,25]. The ratios of antimicrobial resistant Salmonella isolates from pet dogs were lower than humans, revealing a situation of more antimicrobial resistance among humans than pet dogs. However, the presence of antimicrobial resistance among the Salmonella isolates from both dogs and humans revealed a serious situation of antimicrobial resistance in Khon Kaen, Thailand.

For the epidemiological relatedness results of Salmonella infection among paired dogs and humans, (1) the relationship between pet dogs and their human caregivers for the occurrence of Salmonella infection was not statistically different ( $p=0.310, n=140)$ when analyzed by McNemar's test; (2) the Salmonella serotypes between pet dogs and humans were not significantly different $(p>0.05)$; and (3) the resistance patterns of Salmonella isolates were not significantly different between human and pet dog isolates $(p>0.05)$. This reveals that the presence of Salmonella, the AMR patterns, and the serotypes from pet dogs and humans were quite similar. However, the number of homologous Salmonella isolate pairs (the cases that were infected between each other or from similar sources) among the total Salmonella-present households was only 5.13\%, indicating that the assumption of high risk close contact infection between pet dogs and their caregivers [7-9] was doubtful. The data suggests that close contact between pet dogs and humans was not the main cause of Salmonella infection among humans in Khon Kaen, Thailand (the true rate of close contact should be equal to or lower than the rate of the homologous Salmonella isolate pairs). This conclusion is consistent with the research that household pet dogs as a source of Salmonella was less important than other exposures [6]. The reason for the similar Salmonella presence, AMR patterns, and serotypes with a low proportion of homologous Salmonella isolate pairs, is possibly due to the similar living environments between pet dogs and human caregivers but a low risk of infection passage between the two populations. To obtain credible information on the risk of pet dogs as a source AMR Salmonella, more research on the evaluation of other areas are needed as living styles vary by region.

In this study, paired sampling was applied in the investigation of the epidemiological association of AMR Salmonella between dogs and their caregivers. The idea came from noting the role of AMR bacteria transmission among animals and humans, which may play an important part in the AMR problem, in which blocked critical points may reduce AMR bacteria transmission [1,2]. However, there is limited data on the transmissibility of AMR bacteria among humans. To explore the risk of the critical points of AMR bacteria transmission, the epidemiological association of AMR Salmonella infections between pets and their caregivers (i.e., the close contact way for AMR Salmonella spread) in Khon Kaen was investigated in this work. This paired sample investigation seems like a variant of the cross-sectional study. The variables of a cross-sectional study are measured at a single time. It comprises the measurement of exposure and/or outcome. Traditional cross-sectional studies benefit by assessing the burden of disease in a population, examining the trends of disease, and comparing the disease prevalence of different populations [26]. The paired sample investigation was carried out on 
two matched populations. It provided more information on the differences and similarities compared to traditional cross-sectional studies. This work benefited from the use of paired samples, in which the low proportion of homologous Salmonella isolate pairs revealed that close contact between dogs and caregivers was not a major factor of human AMR Salmonella infections in Khon Kaen, Thailand, which proved to be a counterexample for the assumption of the high risk of close contact infection between dogs and their caregivers [7-9]. Lastly, this work was limited in: (1) the ability to confirm the Salmonella transmission route among the study participants (e.g., the homologous Salmonella isolate pairs may have been due to transmission of infection between the dog and caregivers, or the infection may have been due to transmission to each individual by the same source and therefore not from each other); (2) the low quantity of pet dog source AMR Salmonella evaluations from other regions; (3) not having available symptomatic pet dogs and humans with diarrhea which could have influenced the results.; (4) determining a higher confidence level of the Salmonella prevalence among the study participants due to the limitations of bacterial cultures.

\section{Conclusions}

In this study, the presence of similar Salmonella isolates, AMR patterns, and serotypes with a low proportion of homologous Salmonella isolate pairs was found, possibly due to similar living environments among pet dogs and human caregivers. Moreover, it was determined that a low proportion of AMR Salmonella infections between pet dogs and humans out of the total Salmonella-present households was related to each other. Thus, close contact between pet dogs and their human caregivers might not be the major factor for Salmonella infections or the spread of AMR Salmonella in Khon Kaen, Thailand. More Salmonella research is needed to include studies with larger sample sizes and higher Salmonella detection methods to determine the presence, prevalence, distribution and methods of transmission.

Author Contributions: Conceptualization, X.W. and F.S.; Data curation, X.W.; Formal analysis, X.W., A.K. and S.S.; Funding acquisition, F.S. and S.A.; Investigation, X.W., A.K. and S.K.; Methodology, X.W., C.P., F.S. and S.A.; Project administration, F.S. and S.A.; Resources, S.A.; Software, X.W.; Supervision, F.S.; Validation, F.S. and S.A.; Visualization, X.W.; Writing—original draft, X.W.; Writing—review and editing, F.S. and A.L.R. All authors have read and agreed to the published version of the manuscript.

Funding: This research and the APC were funded by Khon Kaen University Graduate Study Fund, Vet Products Group, and Unovet Network Co. Ltd.

Acknowledgments: This study was conducted with financial support from the Faculty of Veterinary Medicine, Khon Kaen University, Thailand. The authors would like to thank the WHO National Salmonella and Shigella Center (NSSC), Thailand and the Synchrotron Light Research Institute (Public Organization), Thailand for their help.

Conflicts of Interest: The authors declare no conflict of interest.

\section{References}

1. Huddleston, J.R. Horizontal gene transfer in the human gastrointestinal tract: Potential spread of antibiotic resistance genes. Infect Drug Resist. 2014, 7, 167-176. [CrossRef] [PubMed]

2. World Health Organization (WHO). Available online: https://www.who.int/en/news-room/fact-sheets/detail/ salmonella-(non-typhoidal) (accessed on 26 March 2020).

3. Divek, V.T.N.; Venkitanarayanan, K.; Kollanoor, A. Antibiotic-resistant Salmonella in the food supply and the potential role of antibiotic alternatives for control. Foods 2018, 7, 167-291.

4. Aarestrup, F.M.; Hendriksen, R.S.; Lockett, J.; Gay, K.; Teates, K.; McDermott, P.F.; Gerner-Smidt, P. International spread of multidrug-resistant Salmonella Schwarzengrund in food products. Emerg. Infect Dis. 2007, 13, 726-731. [CrossRef] [PubMed]

5. Majowicz, S.E.; Musto, J.; Scallan, E.; Angulo, F.J.; Kirk, M.; O’Brien, S.J.; Jones, T.F.; Fazil, A.; Hoekstra, R.M. The global burden of nontyphoidal Salmonella gastroenteritis. Clin. Infect Dis. 2010, 50, 882-889. [CrossRef] [PubMed] 
6. Christidis, T.; Hurst, M.; Rudnick, W.; Pintar, K.D.M.; Pollari, F. A comparative exposure assessment of foodborne, animal contact and waterborne transmission routes of Salmonella in Canada. Food Control. 2020, 109. [CrossRef]

7. Centers for Disease Control and Prevention (CDC). Available online: https://www.cdc.gov/salmonella/pettreats-07-19/ (accessed on 26 March 2020).

8. Overgaauw, P.A.M.; Vinke, C.M.; Hagen, M.; Lipman, L.J.A. A One Health Perspective on the Human-Companion Animal Relationship with Emphasis on Zoonotic Aspects. Int. J. Environ. Res. Public Health 2020, 17, 3789. [CrossRef] [PubMed]

9. Polpakdee, A.; Angkititra, S.; Suksawat, F.; Sparagano, O.; Kanistanon, K. Epidemiology and antimicrobial resistance of Salmonella sp. isolated from dogs and cats in northeastern Thailand. J. Anim. Vet. Adv. 2012, 11, 618-621. [CrossRef]

10. Da Costa, P.M.; Loureiro, L.; Matos, A.J. Transfer of multidrug-resistant bacteria between intermingled ecological niches: The interface between humans, animals and the environment. Int. J. Environ. Res. Public Health 2013, 10, 278-294. [CrossRef] [PubMed]

11. World Health Organization (WHO). Estimates of the Global Burden of Foodborne Disease. Available online: https://extranet.who.int/sree/FERG/FERG_DataFiles.zip (accessed on 26 March 2020).

12. Sattaburuth, A. Stray Dogs Set to Reach One Million. 2016. Available online: https://www.bangkokpost.com/ thailand/general/944169/stray-dogs-set-to-reach-one-million (accessed on 26 March 2020).

13. Sirinavin, S.; Pokawattana, L.; Bangtrakulnondh, A. Duration of nontyphoidal Salmonella carriage in asymptomatic adults. Clin. Infect Dis. 2004, 38, 1644-1645. [CrossRef] [PubMed]

14. International Organization for Standardization (ISO). Microbiology of Food and Animal Feeding Stuffs-Horizontal Method for the Detection of Salmonella Spp; ISO Norm 6579; International Standardization Organization: Geneva, Switzerland, 2002.

15. Grimont, P.A.; Weill, F.X. Antigenic Formulae of the Salmonella Serovars. WHO Collaborating Center for Reference and Research on Salmonella, 9th ed.; Institut Pasteur Paris: Paris, France, 2007.

16. Clinical Laboratory Standards Institute (CLSI). Performance Standard for Antimicrobial Susceptibility Testing Twenty-Fourth Informational Supplement M200-S24; CLSI: Wayne, PA, USA, 2014.

17. MÄNNIG, A.; Baldauf, N.A.; Rodriguez-Romo, L.A.; Yousef, A.E.; Rodriguez-Saona, L. Differentiation of Salmonella enterica serovars and strains in cultures and food using infrared spectroscopic and microspectroscopic techniques combined with soft independent modeling of class analogy pattern recognition analysis. J. Food Prot. 2008, 71, 2249-2256. [CrossRef] [PubMed]

18. Davis, R.; Mauer, L.J.C. Fourier transform infrared (FT-IR) spectroscopy: A rapid tool for detection and analysis of foodborne pathogenic bacteria. Curr. Res. Top Appl. Microbiol. Microb. Biotechnol. 2010, 2, 1582-1594.

19. Centers for Disease Control and Prevention (CDC). Standard Operating Procedure for Pulsenet PFGE of Escherichia Coli O157: H7, Escherichia Coli Non-O157 (STEC), Salmonella Serotypes, Shigella Sonnei and Shigella Flexneri; Department of Health and Human Services: Atlanta, GA, USA, 2013.

20. Kiflu, B.; Alemayehu, H.; Abdurahaman, M.; Negash, Y.; Eguale, T. Salmonella serotypes and their antimicrobial susceptibility in apparently healthy dogs in Addis Ababa, Ethiopia. BMC Vet. Res. 2017, 13, 134. [CrossRef] [PubMed]

21. Reimschuessel, R.; Grabenstein, M.; Guag, J.; Nemser, S.M.; Song, K.; Qiu, J.; Pabilonia, K. Multilaboratory survey to evaluate Salmonella prevalence in diarrheic and nondiarrheic dogs and cats in the United States between 2012 and 2014. J. Clin. Microbiol. 2017, 55, 1350-1368. [CrossRef] [PubMed]

22. Hendriksen, R.S.; Bangtrakulnonth, A.; Pulsrikarn, C.; Pornruangwong, S.; Noppornphan, G.; Emborg, H.D.; Aarestrup, F.M. Risk factors and epidemiology of the ten most common Salmonella serovars from patients in Thailand: 2002-2007. Foodborne Pathog. Dis. 2009, 6, 1009-1019. [CrossRef] [PubMed]

23. Angkititrakul, S.; Chomvarin, C.; Chaita, T.; Kanistanon, K.; Waethewutajarn, S. Epidemiology of antimicrobial resistance in Salmonella isolated from pork, chicken meat and humans in Thailand. Southeast Asian J. Trop. Med. Public Health. 2005, 36, 1510-1515. [PubMed]

24. Chotinun, S.; Rojanasthien, S.; Unger, F.; Tadee, P.; Patchanee, P. Prevalence and antimicrobial resistance of Salmonella isolated from carcasses, processing facilities and the environment surrounding small scale poultry slaughterhouses in Thailand. Southeast Asian J. Trop. Med. Public Health. 2014, 45, 1392-1400. 
25. Patchanee, P.; Tansiricharoenkul, K.; Buawiratlert, T.; Wiratsudakul, A.; Angchokchatchawal, K.; Yamsakul, P.; Yano, T.; Boonkhot, P.; Rojanasatien, S.; Tadee, P. Salmonella in pork retail outlets and dissemination of its pulsotypes through pig production chain in Chiang Mai and surrounding areas, Thailand. Prev. Vet. Med. 2016, 130, 99-105. [CrossRef]

26. Aggarwal, R.; Ranganathan, P. Study designs: Part 2-Descriptive studies. Perspect. Clin. Res. 2019, 10, 34-36. [CrossRef] [PubMed] 